\title{
Impact of asbestos on public health: a retrospective study on a series of subjects with occupational and non-occupational exposure to asbestos during the activity of Fibronit plant (Broni, Italy)
}

\author{
Silvia D. Visonà, ${ }^{1}$ Simona Villani, ${ }^{2}$ Federica Manzoni, ${ }^{2}$ Yao Chen, ${ }^{1}$ Giorgio Ardissino, ${ }^{1}$ \\ Francesca Russo, ${ }^{1}$ Matteo Moretti, ${ }^{1}$ Gulnaz T. Javan, ${ }^{3}$ Antonio Osculati ${ }^{1}$ \\ ${ }^{1}$ Section of Legal Medicine and Forensic Science, and ${ }^{2}$ Section of Biostatistics and Clinical Epidemiology, \\ Department of Public Health, Experimental and Forensic Medicine, University of Pavia; ${ }^{3}$ Forensic Science \\ Program, Department of Physical Sciences Alabama State University, Montgomery, AL, USA
}

\section{Significance for public health}

The widespread production and use of asbestos have caused unprecedented human suffering and still represents a major public health problem all over the world. The particularly prolonged latency of the disease has led to the onset of a catastrophic epidemics affecting people who suffered exposure even decades ago. Even though the use of asbestos has been banned in Europe, as well as in the US, in many countries it is still allowed (e.g. Russia, Kazakhstan, Brazil, China). Asbestos is still widely used for insulation, house construction and shipbuilding, and still represents a big business. The diseases caused by asbestos can be divided in two main groups: the non-neoplastic diseases, such as the benign manifestation known as pleuric plaques and, on the other hand, asbestosis, related to the absorption of high amounts of asbestos fibers. The second group includes the malignant neoplastic diseases: lung cancer and mesothelioma. Mesothelioma has to be considered of particular importance, even though its incidence is extremely low in general population, causing, on a global scale, about the $1 \%$ of deaths due to tumors. The extreme relevance of this disease, and consequently the great need of research in this field, is owed to its known relationship with a well-defined trigger (asbestos) and its exceptionally poor prognosis.

\footnotetext{
Abstract

The goal of this study is to understand more about the role of asbestos in causing human diseases, first of all mesothelioma, by investigating a large series of deaths due to asbestos-related diseases (ARDs). The main aim is to clarify if even very low amounts of asbestos can cause mesothelioma and other ARDs, as well as to find out if a different individual vulnerability can be important. This retrospective study included 188 subjects who died from asbestos related diseases in 2000-2017 in the area around Broni, Italy, where an important asbestos cement factory had been active from 1932 until 1993. In each case, a forensic autopsy has been performed. In order to perform the present study, the records were retrieved, including the clinical files, the autopsy, and the histological report. The statistical analysis performed showed that there was a significant relation between the cause of death (mesothelioma, lung cancer or asbestosis) and the kind of exposure (occupational, neighborhood or household), showing that all the subjects not exposed occupationally (and, therefore, exposed to lower amounts of asbestos) died from mesothelioma, whereas the individuals who used to work at the plant died also from other caused (asbestosis, lung cancer). Significant
}

differences were highlighted examining the distribution of the causes of death according to the smoking habits. Moreover, among the mesothelioma patients, the survival time was shorter in the subjects with a neighborhood or household exposure than in the occupationally exposed individuals. The study provided meaningful data about the role of asbestos in causing human pathologies. In particular, the present data appear to support the hypothesis that even an exposure to a very little amount of asbestos can cause mesothelioma in hypersusceptible subjects (probably, on a genetic basis).

\section{Introduction}

Asbestos (from the Greek word meaning "inextinguishable") is the term for a family kind of naturally occurring minerals that readily separate into thin fibers and are found in many parts of the world abundant and widespread in nature. ${ }^{1}$

Asbestos fibers are classified into two main groups of silicates: serpentine asbestos (that includes only chrysotile, (also called white asbestos), and the vast category of amphiboles, that includes commercial asbestos - crocidolite (also known as blue asbestos), amosite (fibrous-asbestiform variety of grunerite, (also named brown asbestos), and anthophyllite, - as well as the noncommercial asbestos types- tremolite asbestos, and actinolite asbestos.

Chrysotile, crocidolite, amosite, and antophyllite asbestos are exploited in numerous commercial and industrial settings owing to their chemical, physical and technological properties.

Chrysotile accounts for some $90 \%$ to $95 \%$ of all the asbestos used worldwide. Crocidolite and amosite made up the bulk of the commercially used asbestos that was not chrysotile.

There is an open debate about the different hazardousness and cancerogenic potential of the various type of asbestos, ${ }^{2-5}$ according to the World Health Organization (WHO) and the International Agency for Research on Cancer (IARC), all types of asbestos are classified as Class I Carcinogens. ${ }^{1}$ Nevertheless, whether chrysotile by itself can cause mesothelioma remains one of the most controversial issues, as there are opposite opinions in literature. ${ }^{4,5}$ The widespread production and use of asbestos have caused unprecedented human suffering and still represents a major public health problem all over the world. Even though the use of asbestos has been completely banned in Europe since 1999, as well as in the most countries of the world, the WHO estimates that 
125 million people are exposed to asbestos in the workplace. ${ }^{6}$ Worldwide production of asbestos declined between 1980 and 2010 , but on a global scale asbestos is still used, despite the increased recognition of its health consequences. Even though the United States Government has not banned asbestos, its mining stopped in 2002 and its use by industry has been strongly reduced because of a combination of regulation and litigation. However, asbestos exposures in the United States and Western countries still occur from demolition and maintenance of buildings and asbestoscontaining materials.

Canada represents a specific situation. Recently in 2018, the Canadian government passed the Prohibition of Asbestos and Products Containing Asbestos Regulations, sponsored by Environment and Climate Change Canada and Health Canada; however, certain uses of asbestos remain legal in Canada: for instance, nuclear energy industries and Canadian military facilities are allowed to use asbestos through 2029 . The chlor-alkali industry has until 2030 to phase out asbestos. ${ }^{7}$

Asbestos is still a big business in many countries. The high demand for inexpensive piping and roofing material is the driving force behind the business. The world leaders in asbestos production for 2015 and 2016 were Russia, China, Brazil, Kazakhstan and India, according to a 2017 report from the U.S. Geological Survey. Brazil announced a ban of asbestos in 2017. One of the largest asbestos consumers is Russia. Although the country banned only the amphibole type of asbestos in 1999, today it supplies $60 \%$ to $75 \%$ of all asbestos used worldwide. ${ }^{8}$

Many efforts have been made to predict the future course of the mesothelioma epidemic in several European countries. ${ }^{9}$ These analyses have all indicated that, in several countries, the mesothelioma epidemic will not peak for a few more years. In Europe, the peak is predicted to occur between 2010 and $2023 .{ }^{9}$ Predicting the future course of the asbestos epidemic in developing countries is almost impossible due to the lack of evidences about exposures and disease occurrences from many areas of the world. ${ }^{9}$

The latent onset of disease from exposure has led to a catastrophic epidemic and a continuing onslaught as a result of exposures which took place decades ago.

The link between asbestos exposure and Asbestos Related Diseases (ARDs: mesothelioma, lung cancer and asbestosis) is well known. The first epidemiologic study of asbestos textile workers, published by Merewether and Price (1930), both with the UK Government Home Office, established general causation between asbestos exposure and the lung disease, asbestosis. ${ }^{9}$ The knowledge that asbestos is carcinogenic, and the subsequent awareness of the manufacturers, is a crucial concern when it comes to ethics and court litigation. The scientific community is aware of the link between asbestos and mesothelioma since 1960, when Wagner reported the results of a South African study. ${ }^{10}$ Yet, much evidence about the hazardousness of asbestos was available before that time. Above all, it is of great importance to establish when a company known or should have known about the hazards associated about asbestos. It has been proven that, even by the 1930s, industrial facilities used to have the knowledge that exposure to asbestos causes asbestosis. Internal documents located in corporate files revealed that many companies, in the US, not only had the knowledge of the hazard of asbestos but also took active measures to prevent publication or mention of asbestosrelated hazards. ${ }^{11}$

At least since the $1960 \mathrm{~s}$, there are no doubts that asbestos causes two main group of pathologies: the first group is represented by the non-neoplastic diseases, such as the benign manifestation known as "pleuric plaques" and also, asbestosis, the form of pneumoconiosis related to the absorption of high amounts of asbestos fibers. ${ }^{11}$ The second group includes the malignant neoplastic diseases: lung cancer and mesothelioma, the malignant neoplasm arising from the serosal linings of the pleural, pericardial or peritoneal cavities.

Among ARDs, mesothelioma has to be considered of particular importance, even though its incidence is extremely low in general populations, causing on a global scale, approximately $1 \%$ of deaths due to tumors. ${ }^{11}$ The extreme relevance of this disease, and consequently the great need of research in this field, is owed to its known relationship with a well-defined trigger (asbestos) and its exceptionally poor prognosis. Indeed, there is significant variation with important prognostic variables such as age, stage at presentation and histologic subtype, median survivals for patients with mesothelioma generally range from 6-14 months. ${ }^{12}$ Currently, even though several important progresses have been made recently in the care of patients, there are no effective therapies available for mesothelioma.

Despite the relation between asbestos and mesothelioma, lung cancer and asbestosis is well documented, many health issues concerning the etiopathogenesis of ARDs, especially mesothelioma, are still debated. Most importantly, not all cases of mesothelioma are related to a documented exposure to asbestos, occupational or neighborhood (also defined as "environmental"). It is reported that about $20-30 \%$ of mesothelioma patients have never been exposed to asbestos. ${ }^{13}$ On the other hand, the incidence of this disease among occupationally exposed workers varies between $0.5 \%$ and $18 \% .{ }^{14}$ These facts seem to indicate that the neoplasm, similarly to others, can be consequential to triggers other than asbestos. However, asbestos fibers had been found even in lungs of affected subjects exposed to very low doses of asbestos (or not supposed to be exposed at all), suggesting that even very few fibers, in a hypersusceptible individual, might cause mesothelioma. Many attempts have been made in order to identify the substrate to individual vulnerability of mesothelioma.

Up to now, $B A P 1$ is the only gene with a role in determining individual vulnerability to mesothelioma is known. ${ }^{14} B A P 1$ has several cell-intrinsic tumor suppressive functions, such as regulation of cell cycle and replication, gene transcription, DNA damage response, as well as a modulation in the inflammatory response to crocidolite. ${ }^{14}$ Additionally, two genome-wide association studies (GWASs) on mesothelioma were conducted. ${ }^{15,16}$ None of them identified a single gene or even a signature of genes associated to an individual vulnerability to mesothelioma. Therefore, the existence of a predisposing factor to mesothelioma, even though strongly suggested by the scientific evidence so far available, is still an unanswered question.

The goal of the present study is to better understand the consequences of exposure to asbestos, how and to which extent it contributes in causing ARDs, focusing, particularly, on mesothelioma, by investigating, retrospectively, all the cases of asbestos related diseases (ARDs) that underwent a forensic investigation at the Section of Legal Medicine and Forensic Sciences of University of Pavia.

\section{Materials and Methods}

\section{Setting and study design}

This retrospective study includes 188 subjects who died from ARDs in 2000-2017. Almost all of them formerly worked and/or lived in Broni, Italy, a small town located in the Po Valley (northern of Italy) where an important asbestos-cement factory 
named Fibronit had been active from 1932 until 1993, producing asbestos-cement artifacts, using mixtures of commercial types of asbestos, including chrysotile, crocidolite, and amosite (used in small quantities, as an additive). Processes that produced diffuse emissions from building openings and local exhausts were not installed until the end of the 1970s. The records of the plant reported a total number of 3455 workers employed between 1932 and 1993.

Epidemiological studies showed that a large number of cases occurred among subjects never employed in asbestos cement production and the mesothelioma risk increased with residential proximity to the plant. ${ }^{17-19}$

A forensic autopsy was performed for every case of the present study. The records were retrieved, including the clinical files, the autopsy, and the histological report. Then, the dataset was built, including the following variables: age at death, sex, residency, job, the cause of death (if mesothelioma, also the histological type and immunohistochemistry results were included), the kind of exposure to asbestos (occupational, neighborhood, or household), the survival time since the diagnosis, the latency time (defined as the time between the first exposure and the clinical manifestation of the disease), and smoking habit.

\section{Statistical analysis}

Quantitative variables were summarized by mean and standard deviation (SD), otherwise with median and interquartile range (IQR). Qualitative variables were described with absolute frequencies and percentages. Linear and logistic regression models, uni- and multivariable, were applied to adjust the estimates with respect to individual factors (sex, age, profession) and asbestos exposure.

The survival analysis was performed by applying the KaplanMeier estimator, or Taron-Ware if it was appropriate to the specific problem. The association with sex and the type of exposure was analyzed with the Cox model of proportional hazards, and the applicability assumption was evaluated by the Schoenfeld test. The association was expressed as Hazard Ratio (HR) with the corresponding 95\% Confidence Intervals. A P-value $\leq 0.05$ was considered significant. In all test a P-value $\leq 0.05$ has been considered significant. The analyses were conducted using STATA 14 program.

\section{Results}

The study sample was composed of 188 cases, $66 \%$ males. The average age at death was 71.4 years, significantly lower in females than males (Table 1). Almost the totality of the subjects used to live in the province of Pavia at the time of death $(97.3 \%)$.
Regarding the kind of asbestos exposure, the subjects was subgrouped into three categories: occupational, neighborhood, household. In several cases, the subjects had more than one source of exposure. At least $84 \%$ of the entire study group had environmental exposure to asbestos, approximately $27 \%$ household and $60 \%$ occupational. Considering only the group of individuals exposed occupationally, $70 \%$ of the 110 individuals for whom asbestos occupational exposure was ascertained were Fibronit workers and in almost all cases they were males (71/77). For 70 Fibronit workers it was impossible to know the exact period of time in which they worked at the plant, which an average result of approximately 20 years, with a variability of about 10 years.

As expected, based on the strong excess of men employed at Fibronit plant, the frequency of occupational exposure was significantly different between the two genders, as shown in Table 1. Similarly, among women, the frequency of household exposure to asbestos, linked to a family member exposed occupationally, was two times higher than that found among men and this excess is again significant. Instead, the difference between men and women in respect to environmental exposure was not significant (Table 1).

Table 1. The classification of exposure to asbestos according to gender.

\begin{tabular}{cccc} 
& \multicolumn{2}{c}{ Gender } & Statistical test \\
& & Female & p-value \\
Occupational Exposure & $(\mathrm{n}=120)$ & $(\mathrm{n}=63)$ & $\mathrm{Chi}^{2}=72.1$ \\
No & $17.5 \%$ & $82.5 \%$ & $\mathrm{P}<0.001$ \\
Yes & $82.5 \%$ & $17.5 \%$ & \\
Household exposure & $(\mathrm{n}=79)$ & $(\mathrm{n}=58)$ & $\mathrm{Chi}^{2}=8.2$ \\
No & $82.3 \%$ & $60.3 \%$ & $\mathrm{P}<0.004$ \\
Yes & $17.7 \%$ & $39.7 \%$ & \\
\hline Neighborhood exposure $(\mathrm{n}=112)$ & $(\mathrm{n}=63)$ & $\mathrm{Chi}{ }^{2}=3.07$ \\
No & $19.6 \%$ & $9.5 \%$ & $\mathrm{P}=0.08$ \\
Yes & $80.4 \%$ & $90.5 \%$ & \\
\hline \multicolumn{4}{c}{} \\
\hline
\end{tabular}

Table 2. The frequency of the causes of the death in the present study.

\begin{tabular}{lcc} 
Cause of death & N. & $\%$ \\
Mesothelioma & 149 & 79.7 \\
Asbestosis & 20 & 10.7 \\
\hline Lung Cancer & 12 & 6.4 \\
Other tumors & 3 & 1.6 \\
\hline Other cardiopulmonary pathologies & 3 & 1.6 \\
\hline
\end{tabular}

Table 3. The distribution of the cause of death, reclassified by type of exposure.

\begin{tabular}{|c|c|c|c|c|c|c|}
\hline \multirow[t]{2}{*}{ Cause of death } & \multicolumn{2}{|c|}{ Occupational exposure, \% } & \multicolumn{2}{|c|}{ Household exposure, \% } & \multicolumn{2}{|c|}{ Neighborhood exposure, \% } \\
\hline & $\begin{array}{c}\text { No } \\
(\mathrm{n}=73)\end{array}$ & $\begin{array}{c}\text { Yes } \\
(n=109)\end{array}$ & $\begin{array}{c}\text { No } \\
(\mathrm{n}=99)\end{array}$ & $\begin{array}{c}\text { Yes } \\
(n=37)\end{array}$ & $\begin{array}{c}\text { No } \\
(\mathrm{n}=28)\end{array}$ & $\begin{array}{c}\text { Yes } \\
(n=146)\end{array}$ \\
\hline Mesothelioma & 100.0 & 67.0 & 85.9 & 100.0 & 82.1 & 81.5 \\
\hline Asbestosis & 0.0 & 17.4 & 9.1 & 0.0 & 10.7 & 8.9 \\
\hline Lung cancer & 0.0 & 11.0 & 5.1 & 0.0 & 7.1 & 6.9 \\
\hline Other tumors & 0.0 & 2.8 & I & l & 0.0 & 1.4 \\
\hline \multirow[t]{2}{*}{ Other cardiopulmonary diseases } & 0.0 & 1.8 & / & / & 0.0 & 1.4 \\
\hline & \multicolumn{2}{|c|}{$\mathrm{P}^{*}<0.001$} & \multicolumn{2}{|c|}{$\mathrm{P}^{*}=0.051$} & \multicolumn{2}{|c|}{$\mathrm{P}^{*}=0.96$} \\
\hline
\end{tabular}

*Fisher's exact test. 


\section{Cause of death and type of exposure}

In the entire study, the most frequent cause of death was pleural mesothelioma, followed by lung carcinoma and asbestosis. In $80 \%$ of cases, the cause of death, revealed by a forensic autopsy, was found to be a mesothelioma. About $11 \%$ of cases died from asbestosis and its consequences and in the remaining cases another related asbestos pathology, as shown in Table 2.

The distribution of the cause of death, reclassified by type of exposure, showed a very significant difference based on occupational exposure (Table 3). Namely, the 109 subjects exposed occupationally died in most cases (about 67\%) from mesothelioma, in $17.4 \%$ of cases from asbestosis, in $11 \%$ of cases from lung carcinoma and in a percentage lower than $3 \%$ from other cancers or cardiopulmonary diseases. On the other hand, the subjects without occupational exposure (73) all died from mesothelioma. This data implies a significant difference, from a statistical point of view, between the causes of death of the subgroups, respectively, with and without occupational exposure.

\section{Relation between cause of death and cigarette smoking}

Considering the subjects for which it was possible to establish their smoking habits (143) it was possible to point out the following findings. Most of the subjects were ex-smokers or smokers at the time of death $(64.6 \%)$, but significant differences between the two sex were pointed out (Chi2=18.0, $\mathrm{P}<0.001)$ : over $50 \%$ of women never smoked, whereas less than the $25 \%$ of men were non-smokers.

Significant differences were highlighted examining the distribution of the causes of death according to the smoking habits. Over $90 \%$ of non-smokers died from mesothelioma. The same was observed regarding smokers. On the contrary, only $69 \%$ of exsmokers died from mesothelioma, whereas, the $13 \%$ and $15,6 \%$ of them died from lung cancer and asbestosis, respectively (Table 4). Moreover, it was found that the occurrence of death due to mesothelioma is less frequent among ex-smokers in the occupational exposed subgroup compared to the ex-smokers without such exposure. In addition, a significant relation was found between cause of death and smoking $(\mathrm{P}=0.026)$ in the neighborhood-exposure subgroup: more than $90 \%$ of non-smokers and current smokers died from mesothelioma, while the exsmokers died for this cause less frequently (gap - 20\%). This fact may be due to the very poor prognosis of mesothelioma: the survival after diagnosis is inevitably too short for having time to stop smoking. Among those who did not have environmental exposure, although there was a different distribution of causes of death based on smoking, this is not significant $(\mathrm{P}=0.414)$.

\section{Latency (defined as the time interval between the first exposure and diagnosis) for mesothelioma with respect of gender and type of exposure}

The average latency time between the first neighborhood exposure to asbestos (that corresponds to the date of birth for the subjects who always lived in Broni) and a diagnosis of ARD was about 54 years, slightly higher in men than in women. Limiting the analysis to the subjects who died from mesothelioma (149) it was found that:

I) males had, on average, a latency time from the first environmental exposure higher than the females (55.4 $\pm 13.7 v s$. $52.7 \pm 17.7 \mathrm{yrs})$, but not significantly $(\mathrm{t}=0.88, \mathrm{P}=0.38)$.

II) the latency from the first occupational exposure to asbestos in men was significantly lower than that of women (median time 45 yrs [36-50] vs. 55 yrs [42-58]; $\mathrm{MW}=1.87, \mathrm{P}=0.06$ ) although with statistical significance border line.
Survival time following the diagnosis to mesothelioma, asbestosis, lung cancer with respect of sex and type of exposure

According to this study, the survival since the diagnosis of mesothelioma was significantly higher in men than in women (Tarone-Ware test $=6.92 ; \mathrm{P}=0.0085$ ) as shown in the graph in Figure 1. The median survival time in men is twice higher than in females: 2 years vs. 1 years. Moreover, survival after a diagnosis of mesothelioma is significantly higher in occupationally exposed subjects compared to other kind of exposures (Tarone-Ware Test = 16.49; $\mathrm{P}<0.0001$ ) (Figure 2).

\section{Discussion}

The clinical records have proved to be sufficiently homogeneous, both for sex and for geographical distribution, since almost all the subjects used to live and/or work in the Broni (PV, Italy) area. A source of potential bias has, anyway, to be pointed out: from a statistical point of view, the present series is not comparable to a cohort, as meant in epidemiology, as the subjects, all died for ARDs, where selected only if a forensic autopsy has been performed. This means that the cases here considered have been brought to the attention of the Public Prosecutor (and, therefore, of the Section of Legal Medicine of Pavia University) by physicians who dealt with the case or by the subject's relatives by doctors or others who have come to know about the case. It is

Table 4. The cause of death reclassified according to the cigarette smoking $(\mathbf{P}=\mathbf{0 . 0 1})$.

\begin{tabular}{|c|c|c|c|}
\hline \multirow[t]{2}{*}{ Cause of death } & \multicolumn{3}{|c|}{ Cigarette smoke, $\%$} \\
\hline & $\begin{array}{l}\text { Never } \\
(\mathrm{n}=51)\end{array}$ & $\begin{array}{c}\text { Current } \\
\text { smoker }(n=15)\end{array}$ & $\begin{array}{l}\text { Ex-smoker } \\
\quad(n=77)\end{array}$ \\
\hline Mesothelioma & 92.2 & 93.3 & 67.5 \\
\hline Asbestosis & 5.9 & 6.70 & 13.0 \\
\hline Lung cancer & 0.0 & 0.0 & 15.6 \\
\hline Other tumors & 0.0 & 0.0 & 2.6 \\
\hline Other cardiopulmonary diseases & 2.0 & 0.0 & 1.3 \\
\hline
\end{tabular}

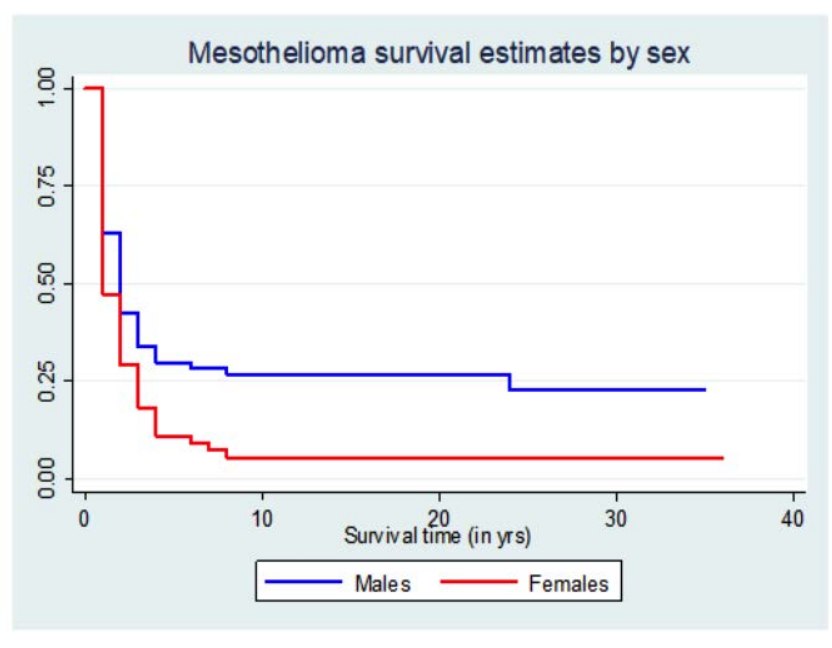

Figure 1. Survival to mesothelioma weighted by sex, assessed using Kaplan-Meier calculator. 
evident obvious that, in case of mesothelioma, a possible link with exposure to asbestos is evident to anyone and, therefore, mesothelioma cases were always reported to the Prosecutor. A similar statement cannot, however, be applied to other diseases, whose relationship with asbestos is less known, such as lung carcinoma or respiratory failure due to pulmonary interstitiopathy.

Notwithstanding, taking this fact into account, the series is suitable to obtain statistically significant and scientifically relevant data.

The concentration of airborne fibers inside a factory where asbestos is processed is much higher than the asbestos detectable in the surrounding environment (subjects with environmental exposure who developed mesothelioma used to live at a distance from Fibronit ranging from 100 meters to 700-1000 meters). Environmental asbestos concentration was measured between 1981 and 1990 (revealing airborne concentrations within the threshold established by current law, with occasional higher values), but never during the 1950s and 1960s, when the factory was fully active and when, considering the long mesothelioma latency, the most relevant exposures probably occurred. ${ }^{18}$

About the exposure, almost the totality of the workers in the asbestos-cement industry were men $(66 \%)$; most of the women included in the sample examined, however, had environmental exposure (they used to live in Broni, nearby the Fibronit plant); a considerable group of the women included in the series had, instead or in addition, a household exposure, as they were in contact with clothes or objects containing asbestos brought into the house by a Fibronit worker.

Even though more than one kind of exposure was pointed out in many subjects, it is obvious that Fibronit workers were exposed to asbestos in such a heavy way; that the neighborhood environmental exposure becomes irrelevant: therefore, in these subjects, occupational exposure was considered predominantly.

Furthermore, among workers in contact with asbestos, although the majority (about 67\%) died from mesothelioma, several subjects did not develop mesothelioma, but instead died for asbestosis (17.4\% of cases) or pulmonary carcinoma (11\%), or, in a percentage lower than $3 \%,(2.8+1.8=4.6)$ for other cancers or cardiopulmonary diseases. However, all 73 cases without occupational exposure, and therefore exposed to low doses of asbestos, died from mesothelioma.

This finding is consistent with other similar reports in literature regarding other plants similar to Fibronit. ${ }^{20,21}$

This data show that we have, on one hand, a considerable number of subjects heavily exposed (Fibronit workers) that, despite having been in contact with a high amount of asbestos, do not develop mesothelioma; on the other hand, there are individuals exposed to very low amounts of asbestos, but anyway develop and die from mesothelioma. This fact might reflect, even in our sample, a different predisposition between individuals, probably explainable on a genetic basis.

However, surprisingly, almost all the women of the sample, despite having been exposed to very low doses of asbestos, died from mesothelioma, compared to men (a large number of them were Fibronit workers), that died also for other causes.

Moreover, interesting data came out from the analysis of survival. In particular, it was observed that survival to mesothelioma was significantly higher in men than in women. One explanation might be that a known occupational exposure allowed a diagnosis at an earlier stage and therefore earlier therapies. But, given the very poor prognosis of mesothelioma and the absence, even nowadays, of effective therapy, it seems more probable that in women (that represent the subjects exposed to a lower dose) the disease has a more serious and aggressive progress. This fact might, again, suggest a genetic predisposition in people exposed to a much lower amount of asbestos who, anyway, developed mesothelioma.

Another issue of great interest, widely discussed in the literature, concerns the very long latency (defined as time between the first exposure and the onset of the pathology) of mesothelioma, that is a unique characteristic of this neoplasm.

The present retrospective study highlighted some interesting evidences: first of all, the average latency time between the first environmental exposure to asbestos and a diagnosis of mesothelioma was about 54 years, slightly higher in men than in women. Asbestosis, on the other hand, tends to occur about 10 years earlier. It must be kept in mind that for many subjects the exposure started at birth, or since the beginning of the productive activities in the factory (1932). It must be kept in mind that for many subjects the exposure started when they were born, or when the activity of the factory started (1932).

Concerning the relation between the latency of mesothelioma and the type of exposure, it seems that the ARDs, overall, appear 10 years before in the subjects exposed occupationally, compared to the other forms of exposure: this could be justified by a stricter medical monitoring, or either by the fact that the amount of asbestos is considerably greater at the workplace and, therefore, the carcinogenic stimulus is repeated and heavier.

Nonetheless, it is scientifically impossible to prove the existence of a safe threshold of exposure and the present data confirm this statement. ${ }^{3}$

As already reported in the previous section, the latency of mesothelioma is particularly protracted, averaging 35-40 years. Latency periods below 20 years must be considered exceptional. ${ }^{13,22}$ Data recently collected in Italy by the organization called "National System for Monitoring Asbestos Diseases" indicate an average latency of 43.6 years, with a DS of 12 and a normal statistical distribution. . $^{23,24}$

Another interesting finding of the present analysis regards the possible contribution of cigarette smoking in causing mesothelioma, in addition to asbestos or independently from it. In our series, smoking does not seem to increase significantly the risk of mesothelioma, and this fits with previous scientific studies. Indeed, the synergistic relationship between cigarette smoking and asbestos in the pathogenesis of lung cancer, as well as in

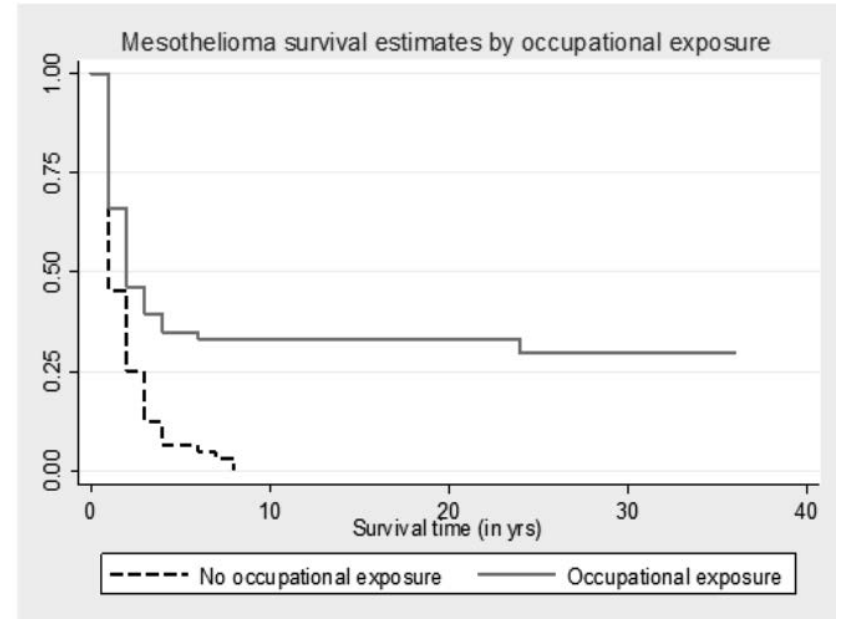

Figure 2. Survival to mesothelioma weighted by occupational exposure, assessed using Kaplan-Meier calculator. 
asbestosis, is well known in the literature, while a significant relation with the pathogenesis of pleural mesothelioma has not been confirmed. ${ }^{25}$ The same can be stated concerning occupational and neighborhood environmental exposure: smoking seems to be completely unrelated from mesothelioma, even in case of very low doses of asbestos.

These data suggest that cigarette smoke cannot be identified as the "missing piece" in the etiopathogenesis of mesothelioma in low exposed individuals.

Overall, it can be concluded that the data above described corroborate the hypothesis, already discussed in literature, that a different individual predisposition to mesothelioma in people exposed to different amounts of asbestos. Therefore, we are encouraged to go ahead investigating this aspect, in order to find out more about the role of asbestos in causing human diseases.

Correspondence: Silvia D. Visonà, Section of Legal Medicine and Forensic Sciences, University of Pavia, Via Forlanini 12, 27100 Pavia.

Tel.: +39.3206056485 - Fax: +39.0382.520025.

E-mail: silviadamiana.visona01@universitadipavia.it

Key words: Asbestos, Mesothelioma, Individual vulnerability.

Contributions: SDV, data collecting, manuscript writing; SV, FM, data analyzing; YC, GA, FR, data collecting; GTJ, A0, manuscript reviewing and references search.

Conflict of interest: The authors declare no potential conflict of interest.

Funding: none.

Received for publication: 8 November 2018.

Revision received: 23 November 2018.

Accepted for publication: 27 November 2018.

(C) Copyright S.D. Visonà et al., 2018

Licensee PAGEPress, Italy

Journal of Public Health Research 2018;7:1519

doi:10.4081/jphr.2018.1519

This work is licensed under a Creative Commons Attribution NonCommercial 4.0 License (CC BY-NC 4.0).

\section{References}

1. Røe OD, Stella GM. Malignant pleural mesothelioma: history, controversy and future of a manmade epidemic. Eur Respir Rev 2015;24:115-31.

2. Hodgson JT, Darnton A. The quantitative risks of mesothelioma and lung cancer in relation to asbestos exposure. Ann Occup Hyg 2000;44:565-601.

3. Gibbs GW, Berry G. Mesothelioma and asbestos. Regul Toxicol Pharmacol 2008;52:S223-31.

4. Smith AH, Wright CC. Chrysotile asbestos is the main cause of pleural mesothelioma. Am J Ind Med 1996;30:252-66.

5. Bernstein DM. The health risk of chrysotile asbestos. Curr Opin Pulm Med 2014;20:366-70.

6. World Health Organization. Asbestos: elimination of asbestosrelated diseases.. Available from: http://www.who.int/newsroom/fact-sheets/detail/asbestos-elimination-of-asbestosrelated-diseases [cited 2018 Nov 3]

7. Mauney M, Pacheco W, Jain SR. Canada - Asbestos Industry, Mesothelioma \& Litigation [Internet]. Mesothelioma Center Vital Services for Cancer Patients \& Families. [cited 2018 Nov 3]. Available from: https://www.asbestos.com/mesothelioma/canada/
8. Mauney M, Pacheco W, Cook JL. Mesothelioma \& Asbestos Use Around the World [Internet]. Mesothelioma Center - Vital Services for Cancer Patients \& Families. [cited 2018 Nov 3]. Available from: https://www.asbestos.com/mesothelioma/worldwide/

9. Stayner L, Welch LS, Lemen R. The worldwide pandemic of asbestos-related diseases. Annu Rev Public Health 2013;34:205-16.

10. Wagner JC, Sleggs CA, Marchand P. Diffuse pleural mesothelioma and asbestos exposure in the North Western Cape Province. Br J Ind Med 1960;17:260-71.

11. Oury TD, Sporn TA, Roggli VL. Pathology of AsbestosAssociated Diseases. Verlag Berlin Heidelberg: Springer; 2014. (Third Edition).

12. Cramer G, Simone CB 2nd, Busch TM, Cengel KA. Adjuvant, neoadjuvant, and definitive radiation therapy for malignant pleural mesothelioma. J Thorac Dis 2018;10:S2565-73.

13. Sekido Y. Molecular pathogenesis of malignant mesothelioma. Carcinogenesis 2013;34:1413-9.

14. Melaiu O, Gemignani F, Landi S. The genetic susceptibility in the development of malignant pleural mesothelioma. J Thorac Dis 2018;10:S246-52.

15. Matullo G, Guarrera S, Betti M, et al. Genetic variants associated with increased risk of malignant pleural mesothelioma: a genome-wide association study. PLoS One 2013;8:e61253.

16. Cadby G, Mukherjee S, Musk AWB, et al. A genome-wide association study for malignant mesothelioma risk. Lung Cancer 2013;82:1-8.

17. Binazzi A, Marinaccio A, Corfiati M, et al. Mesothelioma incidence and asbestos exposure in Italian national priority contaminated sites. Scand J Work Environ Health 2017;43:550-9.

18. Oddone E, Ferrante D, Cena T, et al. [Asbestos cement factory in Broni (Pavia, Italy): a mortality study]. Med Lav 2014;105:15-29.

19. Mensi C, Riboldi L, De Matteis S, et al. Impact of an asbestos cement factory on mesothelioma incidence: global assessment of effects of occupational, familial, and environmental exposure. Environ Int 2015;74:191-9.

20. Kurumatani N, Kumagai S. Mapping the risk of mesothelioma due to neighborhood asbestos exposure. Am J Respir Crit Care Med 2008;178:624-9.

21. Barbieri PG, Mirabelli D, Somigliana A, et al. Asbestos fibre burden in the lungs of patients with mesothelioma who lived near asbestos-cement factories. Ann Occup Hyg 2012;56:66070.

22. Mossman BT, Shukla A, Heintz NH, et al. New insights into understanding the mechanisms, pathogenesis, and management of malignant mesotheliomas. Am J Pathol 2013;182:1065-77.

23. Marinaccio A, Branchi C, Massari S, Scarselli A. National epidemiologic surveillance systems of asbestos-related disease and the exposed workers register. Med Lav 2006;97:482-7.

24. Oddone E, Ferrante D, Tunesi S, Magnani C. Mortality in asbestos cement workers in Pavia, Italy: A cohort study. Am J Ind Med 2017;60:852-66.

25. Geltner C, Errhalt P, Baumgartner B, et al. Management of malignant pleural mesothelioma - part 1: epidemiology, diagnosis, and staging: Consensus of the Austrian Mesothelioma Interest Group (AMIG). Wien Klin Wochenschr 2016;128:611-7. 